Ann. Zootech., I968, 17 (I), 59-7o.

\title{
SUR L'UTILISATION D'UN COMPOSÉ DE FER DEXTRANE HYDROGÉNÉ (1) PAR INJECTION GHEZ LA TRUIE EN GESTATION ET EN LACTATION
}

\author{
M. LELILLJT, I: SALMON-IGTALSELR \\ avec la collaboration technique de Michele Nocart, A. KuOISANT, R. Innier et M. Kinn. \\ Siation de Recherches sur l'Elevage des Porcs, \\ Centre national de Recherches sootechniques, 78 -Jouy-en-Josas \\ Institut national de la Recherche agronomique
}

\section{SOMMAIRE}

Trente truies primipares ont été utilisées pour examiner les possibilités de passage trans-utérin ou trans-mammaire d'un composé de fer injectable. A cet effet, un lot de to truies recevant au $55^{\mathrm{e}}$ jour et au $\mathrm{I}_{\mathrm{O}} \mathrm{e}^{\mathrm{e}}$ jour de gestation une injection intramusculaire de $4 \mathrm{~g}$ de fer dextrane hydrogéné, était comparé à deux autres lots de ro truies ne disposant que d'un régime alimentaire pauvre en fer, supplémenté ou non par 300 p.p.m. de $\mathrm{SO}_{4} \mathrm{Fe}$. Pendant la lactation, la moitié des animaux de chaque lot recevait au ${ }_{1}{ }^{\mathrm{e}}$ jour une nouvelle injection de $4 \mathrm{~g}$ de fer. On a obtenu les résultats suivants.

I. Des injections de fer pendant la gestation accroissent fortement les réserves hépatiques en fer de la 'Truie. Ia diminution du taux d'hémoglobine provocpuée par la gestation est limitée chez les animaux supplémentés. Fin revanche, la répartition du fer et sa teneur dans les productions utérines sont inchangées.

2. La distribution de fer injectable pendant la lactation permet d'accroitre d'environ 5o p. 100 la teneur en fer du lait, mais elle parait insuffisante pour couvrir les besoins des porcelets.

3. La taille et le poids moyen des portées à la naissance ou au sevrage ne sont pas modifiés. On observe chez les porcelets en cours d'allaitement les mêmes symptômes d'anémie quel que soit le traitement effectué sur la mère.

\section{INTRODUCTION}

Il est classique de prévenir l'anémie ferriprive du porcelet sous la mère par une administration orale ou intramusculaire de sels de fer. Un autre procédé consiste à traiter la truie au cours de la gestation ou de la lactation de façon, soit à accroître le stock de fer hépatique du fœutus, soit à obtenir un enrichissement de la teneur en fer du lait maternel. Ceci suppose que le transfert placentaire ou mammaire des sels

(1) Produit commercialisé sous le nom de Fervatol et contenant $100 \mathrm{mg}$ de fer actif et 80 Ul de Vitamine $\mathrm{B}_{12}$ par ml. 
de fer utilisés soit possible. Un certain nombre d'expériences ont été conduites dans ce domaine sur différentes espèces. Leurs résultats sont variables et souvent contradictoires. Fin fait, si le passage du fer à travers le placenta semble se faire assez facilement (RydberG et al., i959 ; Linkeinheimer, I962 ; Koskins et HaNsard, i962, I964), les possibilités d'enrichissement des organes fœtaux sont, par contre, très réduites (RYDBERG et $a l .$, I959; POND, I960; MII I ER et $a l .$, I964). Il en va de même en ce qui concerne l'enrichissement du lait (HAMritor et al., I930; VENx et al., I 947 ; PONd, I960; Chaney et Barnhart, i963; VHum et al., I965). Mais ces phénomènes peuvent varier avec l'espèce considérée, le type de placentation, le stade de gestation et aussi le sel de fer utilisé. Aussi avons-nous entrepris cette étude chez la Truie, en utilisant en injection intramusculaire, du fer dextrane hydrogéné. Ce composé très actif lorsqu'il est utilisé sur les porcelets (LE Dividich et al., r967) présente, contrairement aux autres dextranes de fer, une très grande fluidité, un $\mathrm{pH}$ neutre et une teneur faible en solides totaux qui pourrait en faciliter l'assinilation, la diffusion et le stockage dans les différents organes. Dans notre expérience, nous avons comparé l'influence sur la 'Truie et sa production (contenu utérin, lait) d'un tel traitement, à celle d'un régime alimentaire pauvre en fer, supplémenté ou non par du sulfate ferreux.

\section{MATÉRIEL, ET MÉTHODES}

\section{Animanx}

Trente truies primipares de race Large White appartenant à l'élevage expérimental du C. N. R. Z. ont été utilisées. 'Tous les animaux ont reçu en préexpérience un régime de base pauvre en fer. Ces truies ont été réparties en 3 lots comparables pendant la gestation recevant chacun l'un des trois traitements suivants :

Lot $O$ : régime de base pauvre en fer.

Lot $\mathrm{S}$ : régime de base supplémenté par $\mathrm{SO}_{4} \mathrm{Fe}$ (témoin).

Lot $\mathrm{T}$ : régime de base et 2 injections intramusculaires de $40 \mathrm{ml}$ d'une solution de fer dextrane hydrogéné à $100 \mathrm{mg} / \mathrm{ml}$ chacune, à 55 et 1 Io jours de gestation.

Pendant la lactation toutes les truies ont été soumises au même régime de base pauvre en fer, mais un animal sur deux des lots de gestation a reçu une injection de $40 \mathrm{mI}$ de fer dextrane, 8 jours après la mise bas. I'ensemble des animaux ainsi traité a constitué le lot $\mathrm{T}_{2}$ qui a été comparé au lot $T_{1}$ des animaux sans traitement.

\section{Aliments}

Les différentes matières premières de ces aliments ont toutes été choisies en raison de leur faible teneur en fer (à l'exception du $\mathrm{SO}_{4} \mathrm{Fe}$ ). Les aliments avaient la composition suivante :

\begin{tabular}{|c|c|c|}
\hline & Lots 0 et $\mathrm{T}$ & Lot $S$ \\
\hline$\ldots$ & 40 & 40 \\
\hline Yanioc $\ldots \ldots \ldots \ldots \ldots \ldots \ldots$ & 47 & 47 \\
\hline Lait en poudre .............. & 5 & 5 \\
\hline Rafle de mais broyee............. & 5 & 5 \\
\hline Ifélange minéral sans fer $\ldots \ldots \ldots \ldots$ & 3 & 3 \\
\hline Sulfate de fer..$\ldots \ldots \ldots \ldots \ldots$ & - & 0,03 \\
\hline Teneur en fer (à l'analyse) (p.p.m.)... & 75 & 116 \\
\hline Protéines brutes $(1,100) \ldots \ldots \ldots \ldots$, & 12 & 12 \\
\hline
\end{tabular}




\section{Prélètements et mesures effectuées}

Les mesures suivantes ont été effectuées :

- teneur en hémoglobine et en fer sanguin de la truie, ¿a la saillie, à $60 \mathrm{j}$ de gestation, à la parturition et au sevrage ( $35 \mathrm{j}$ );

- teneur et contenu en fer des organes ou tissus suivants:

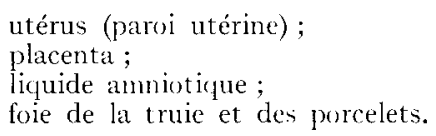

A cette fin, 4 truies de chaque lot étaient sacrifiées an $60^{\mathrm{e}} \mathrm{j}$ de gestation (soit 5 jours après la première injection) et deux porcelets par truie restante étaient sacrifiés à la naissance.

- Teneur en fer du colostrum et du lait de chaque truie à la naissance, à ro j (soit $2 \mathrm{j}$ après la $3^{\mathbf{e}}$ injection) et au sevrage $(35 \mathrm{j})$. A cet effet, on récolt ait par traite manuelle sous ocytocine des échantillons de jo à $100 \mathrm{ml}$ de lait par truie.

- Poids et nombre des porcelets par portée à $60 \mathrm{j}$ de gestation, à la parturition et au sevrage.

- Teneur en hémoglobine du sang des porcelets à la naissance et au sevrage.

- Mortalité embryonnaire et post-natale chez les porcelets.

- Sur le sang :

$$
\text { 4. Dosage }
$$

l'hémoglobine a été dosée par la méthode colorinétrique de KnaLifa, et Salail (I954); le fer total a été déterminé selon la méthode de Ilmanry (1945).

- Sur le lait et le liquide amniotique : le fer total a été dosé par la méthode d'Alifax et Bejandies (I956).

- Sur les tissus (foie, paroi utérine, placenta, porcelet); le fer total a été dosé par la méthode de HENRY (I945) après minéralisation sulfonitrique et oxydation permanganique.

\section{Fixpression des résultats - Analyse statistique}

- Les taux d'hémoglobine sont exprimés en $\mathrm{g}$ p. $100 \mathrm{ml}$ de sang.

- Les teneurs en fer sont exprimées en $\mu \mathrm{g}$ par g de tissu frais.

- La mortalité des porcelets à $60 \mathrm{j}$ de gestation est exprimée en p. roo du nombre de corps jaunes ; à la parturition, en p. Ioo du nombre total des porcelets nés vivants + mort-nés; au sevrage, en p. roo des porcelets nés vivants.

- L'analyse statistique des résultats a été effectuée i l'aide de la méthode descomparaisons multiple des moyennes de DCNCAN (1955).

\section{RÉSUL'TA'TS}

Les tableaux I à 5 résument l'influence des différents traitements sur les truies (tab1. I), les prođuctions utérines (tabl. 2), le lait (tabl. 3), et les porcelets eux-mêmes (tab1. 4 et 5). On peut faire à ce sujet les commentaires suivants.

\section{Taux d'hémoglobine et stockage du fer chez la Truie}

Pendant la gestation, les truies de tous les lots présentent une légère augmentation (avant 60 jours de gestation), puis une diminution de la teneur en hémoglobine de leur sang. Seule cette dernière est significative, mais on peut remarquer qu'elle est sensiblement plus faible dans le lot supplémenté en fer par injection ( $T$ ). 


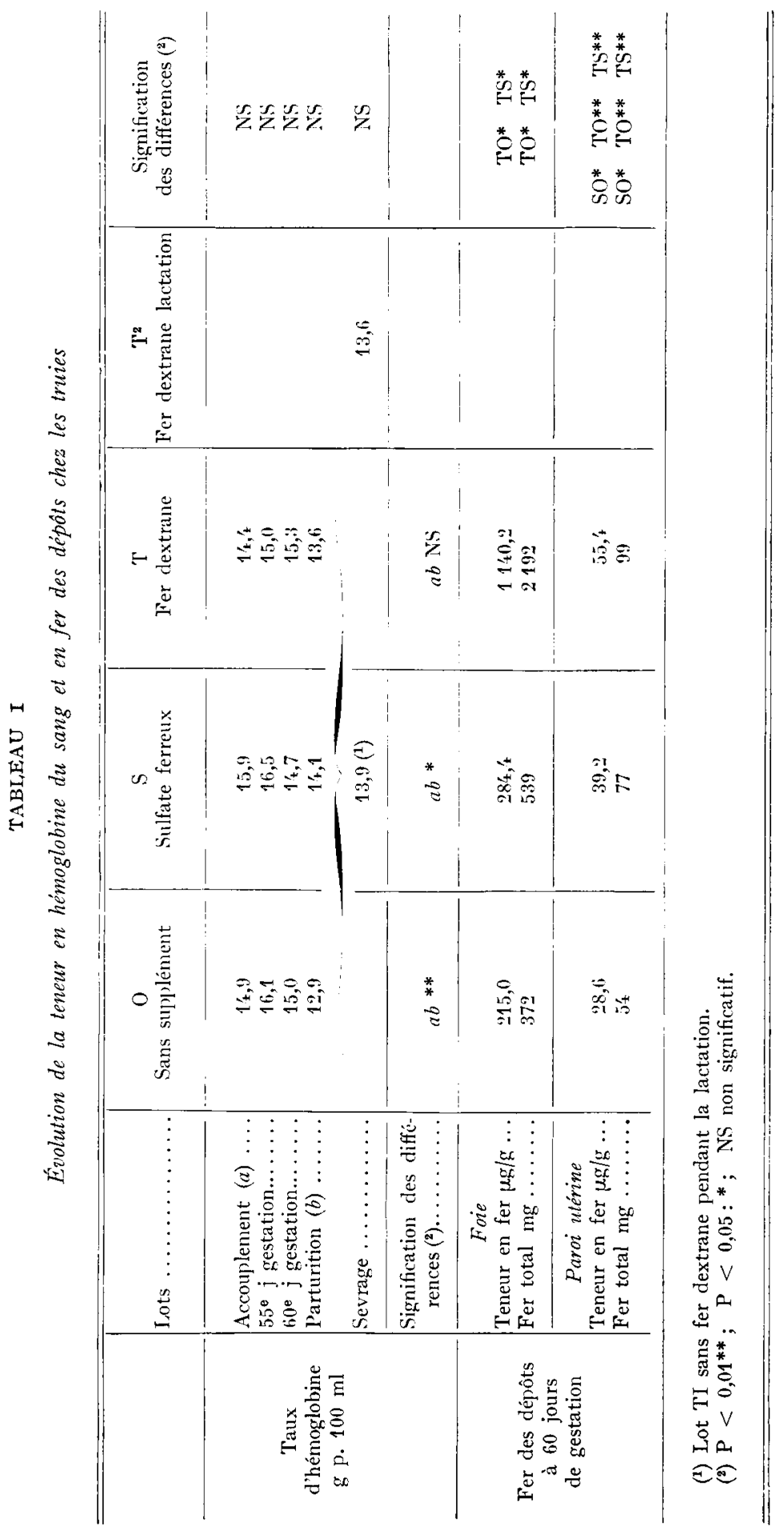


UTILISATION DU FER DEXTRANE PAR LA TRUIE

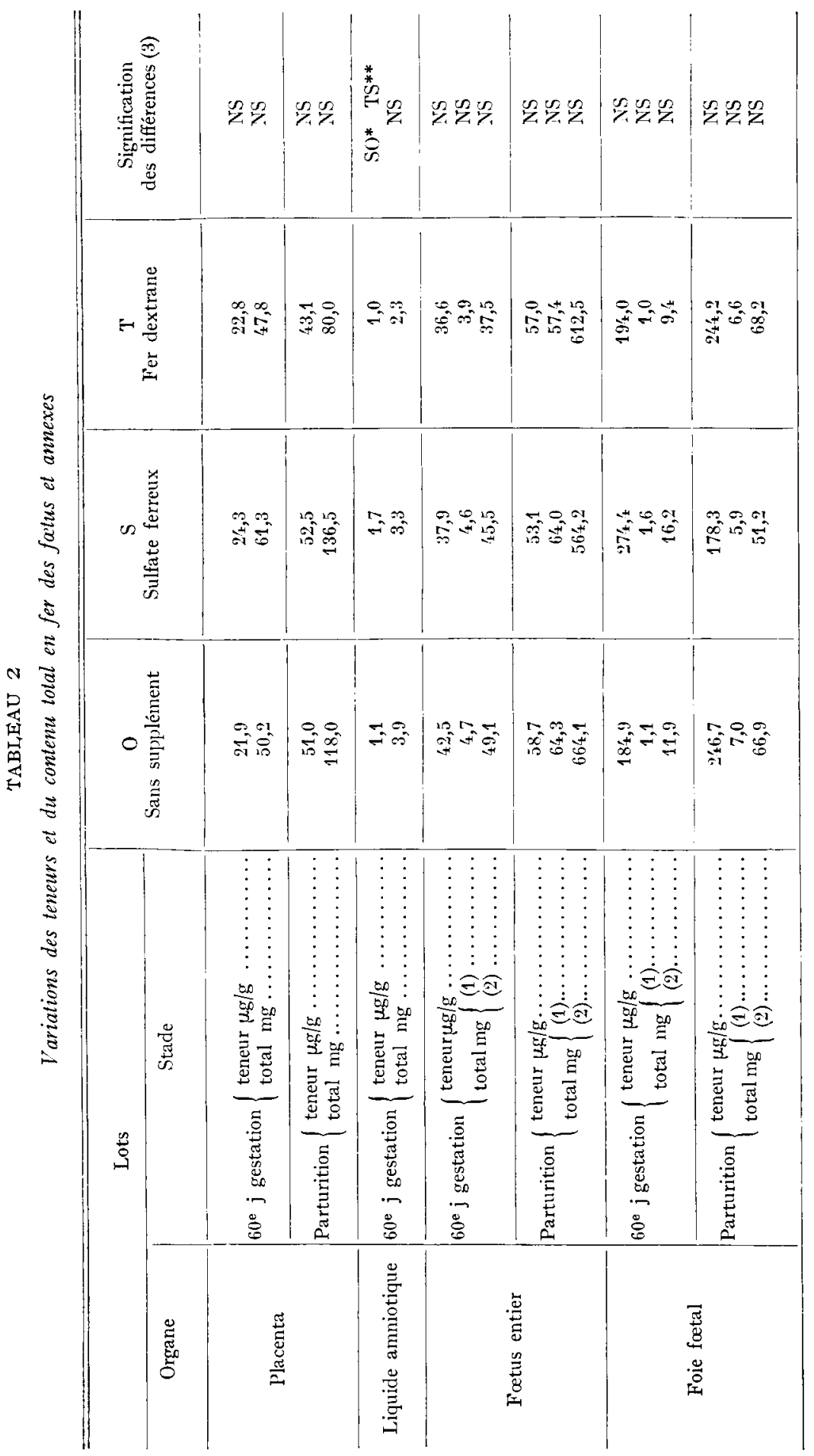

葛

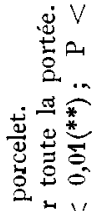
范 डิ중 
Ce dernier a donc été relativement protégé contre 1'appauvrissement du sang en hémoglobine qui apparait classiquement chez les femelles en fin de gestation. Pendant la lactation, le taux d'hémoglobine se maintient, quel que soit le traitement, au niveau acquis en fin de gestation.

Le dépôt du fer chez la 'Truie n'a été déterminé qu'à un seul stade, au $60^{\mathrm{e}}$ jour de gestation, soit 5 jours après la première injection de fer au lot ' $\mathrm{T}$. On remarque cependant, dès ce stade, des différences importantes entre les lots. Les truies du lot recevant du sulfate de fer (S) semblent avoir stocké davantage de fer dans leur foie et dans leur utérus que celles du lot carencé (o). La différence, d'environ $40 \mathrm{p}$. Ioo n'est cependant pas significative. Par contre, chez les truies du lot traité au fer dextrane (T), l'augmentation des dépôts de fer est considérable, et significative. Celle-ci apparaît essentiellement dans le foie, dont le contenu en fer est multiplié par 6 et, accessoirement, dans la paroi de l'utérus dont les réserves sont presque doublées.

TABILAU 3

Évolution de la teneur en fer du lait de Truie

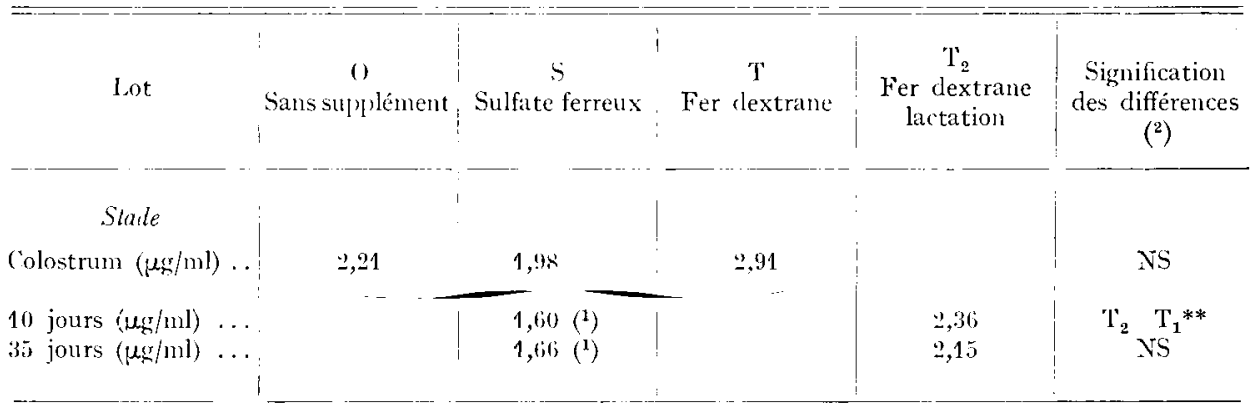

(1) Lot ' $\mathrm{l}_{1}$ sans fer dextrane pendant la lactation.

(2) $\mathrm{l}^{\prime}<0,0 \mathrm{i}^{* *}$.

Par comparaison avec la quantité de fer injectée $(4 \mathrm{~g})$, on remarque que près de 45 p. Ioo de cette dose ont été retrouvés dans le foie et environ ro p. Ioo dans la paroi utérine 5 jours après l'injection.

\section{Productions utérines}

Contrairement aux organes maternels et notamment à la paroi utérine elle-même, les teneurs et les dépôts de fer des compartiments utérins (fœetus, placenta, liquide) ne semblent pas avoir été affectés par les différents traitements (tab1. 2). En particulier, le lot supplémenté par injection (T) ne manifeste aucune richesse particulière en fer au niveau de ces formations. Il semblerait même que la teneur en fer du liquide amniotique et du foie fotal soit un peu plus élevée dans le lot supplémenté en sulfate ferreux. 
UTILISATION DU FER DEXTRANE PAR LA TRUIE

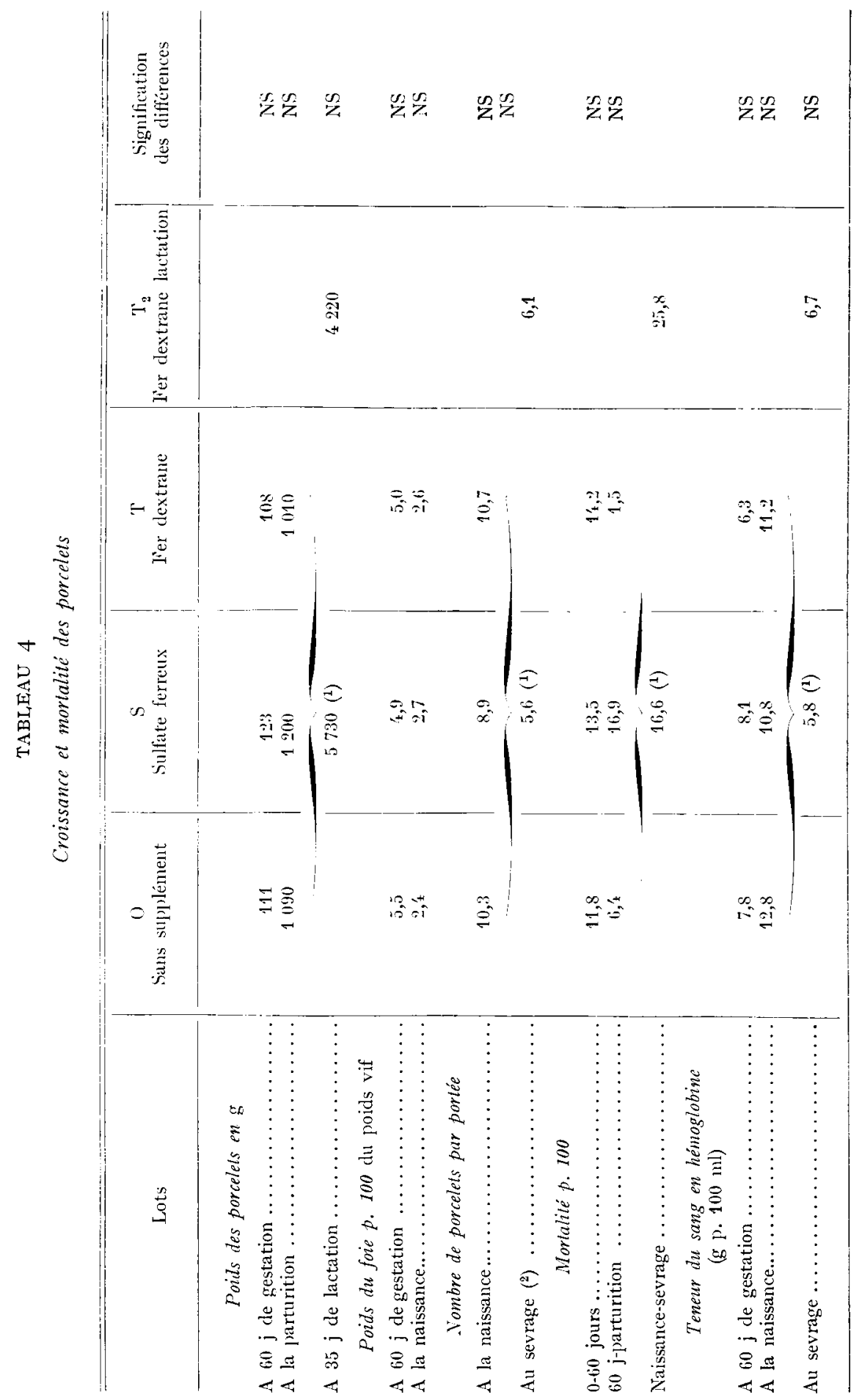


On remarque, par ailleurs, une modification de la répartition du fer au cours de la gestation. En effet, la teneur du placenta double entre les deux stades étudiés tandis que celle du foetus s'accroît moins fortement (environ $40 \mathrm{p}$. Ioo). Mais, comptetenu des variations relatives de poids de ces deux compartiments, le dépôt placentaire, initialement plus important que le dépôt fœetal, s'accroît moins vite que ce dernier. Il en résulte qu'à la parturition, la masse fœetale représente une quantité de fer supérieure à celle des annexes et de la paroi utérine réunies.

TABIEAU 5

Repartition corporelle du fer à la naissanco

\begin{tabular}{|c|c|c|c|c|}
\hline & $\begin{array}{c}\text { O } \\
\text { Sins } \\
\text { stuj]ément }\end{array}$ & $\begin{array}{l}\quad \dot{s} \\
\text { Sulfate } \\
\text { ferreux }\end{array}$ & $\begin{array}{l}\text { 'T' } \\
\text { Fer } \\
\text { dextrane }\end{array}$ & $\begin{array}{l}\text { Signilication } \\
\text { des } \\
\text { différences }\end{array}$ \\
\hline Sang & & & & \\
\hline $\begin{array}{l}\text { T'eneur en fer }(\mathrm{p} .100) \ldots \ldots \ldots \ldots \ldots \ldots \\
\text { Fer sanguin total }(1, \ldots \ldots \ldots \ldots \ldots \ldots \ldots \\
\text { L. } 100 \text { fer total. } \ldots \ldots \ldots \ldots \ldots \ldots \ldots\end{array}$ & $\begin{array}{l}11,0+16 \\
211,1 \\
: 1,2\end{array}$ & $\begin{array}{l}0,1033^{\prime} 6 \\
14,1 \\
31,0\end{array}$ & $\begin{array}{l}0,0: 37: 2 \\
11,0 \\
28,9\end{array}$ & $\begin{array}{l}\mathrm{NS} \\
\mathrm{NS}\end{array}$ \\
\hline $\begin{array}{l}\text { Teneur en fer }(\mu \mathrm{g} / \mathrm{g}) \ldots \ldots \ldots \ldots \ldots \ldots \\
\text { Fer hej } \ldots \text { atique total }(\mathrm{mg}) \ldots \ldots \ldots \ldots \ldots \\
\text { P. } 100 \text { fer total } \ldots \ldots \ldots \ldots \ldots \ldots \ldots \\
\qquad \text { Curcasse }\end{array}$ & $\begin{array}{r}26,7 \\
2,0 \\
11,9\end{array}$ & $\begin{array}{r}17 x,: 3 \\
5,9 \\
11,1\end{array}$ & $\begin{array}{r}21+9 \\
6,6 \\
11,5\end{array}$ & $\begin{array}{l}\mathrm{NS} \\
\mathrm{NS}\end{array}$ \\
\hline 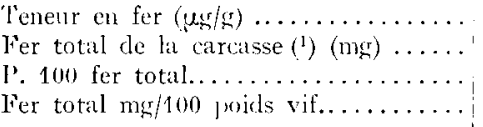 & $\begin{array}{l}: 36,6 \\
: 37,2 \\
37,8 \\
5,2\end{array}$ & $\begin{array}{r}32,9 \\
38,2 \\
59,4 \\
5,9\end{array}$ & $\begin{array}{l}3,5,0 \\
31,3 \\
i ! 3,7 \\
5,7\end{array}$ & $\begin{array}{l}\text { NS } \\
\text { NS }\end{array}$ \\
\hline
\end{tabular}

(1) La carcasse représente le porcelet complet saigné clont le foie al ćté prélevé.

\section{Colostrum et lait}

Le tableau 3 rapporte l'évolution de la teneur en fer de la sécrétion lactée des truies au cours de la lactation. Compte tenu du petit nombre d'observations, les résultats des différents lots ont été regroupés à partir de ro jours selon que les truies avaient reçu (lot $T_{2}$ ) ou non (lot $\left.T_{1}\right)$ une injection de fer à la fin de la première semaine de lactation.

On remarque qu'avant ce stade (colostrum) les différences de richesse en fer sont peu importantes et non significatives entre les lots. Tout au plus trouve-t-on une légère tendance pour le colostrum des truies $T_{1}$ à contenir un peu plus de fer que celui des autres lots, ce qui est à rapprocher de l'augmentation observée dans le foie et l'utérus de ces mêmes truies.

Par contre, les différences deriennent plus nettes au cours de la lactation : on note alors une augmentation significative d'environ $5^{\circ} \mathrm{p}$. roo de la teneur en fer du 
lait chez les truies deux jours après l'injection. Celle-ci semble se maintenir au moins jusqu'à 35 jours, malgré des variations individuelles importantes (différence non significative).

\section{Fatus et porcelets}

Les données se rapportant à la croissance et à la mortalité des porcelets aux différents stades sont rassemblées dans le tableau 4 . I,e tableau 5 indique en outre la répartition corporelle $d u$ fer à la naissance chez les porcelets produits par les truies des 3 lots de gestation.

Aucun de ces critères ne semble faire ressortir la supériorité de l'un quelconque des traitements et notamment des injections de fer faites à la truie avant ou après la parturition ( $\mathrm{T}, \mathrm{T}_{2}$ ).

On remarquera également que le poids des porcelets à 35 jours (sevrage) est relativement faible dans tous les lots (il est habituellement voisin de $8 \mathrm{~kg}$ ). Ce résultat est à rapprocher de la mortalité très élevée qui est apparue dans tous les lots après la naissance et de la chute importante des teneurs en hémoglobine observée pendant l'allaitement (cette chute paraît cependant plus faible pour le lot $\mathbf{T}_{\mathbf{2}}$ ). Ces deux phénomènes permettent de penser qu'une très forte anémie a sévi parmi les porcelets des différents lots (ces derniers ne recevaient en effet aucun traitement antianémique direct).

Il ressort aussi du tableau 5 que les stocks de fer hépatique sont relativement faibles à la naissance quels que soient les lots, en regard du fer total déposé dans l'organisme et notamment dans la carcasse.

\section{DISCUSSION}

Le premier point était de savoir dans quelle mesure le fer dextrane injecté pouvait traverser la barrière placentaire. Notre expérience ne nous permet pas d'y répondre pleinement: il est certain que le fer injecté a remarquablement diffusé dans tout l'organisme de la Truie puisqu'il s'en est trouvé des quantités considérables dans le foie et une fraction non négligeable dans la paroi utérine, résultat comparable à celui trouvé par MORGAN (I96I) sur le Rat. Par ailleurs, le taux d'hémoglobine plus élevé observé à la parturition chez les truies traitées pourrait indiquer la formation d'un dépôt accru de fer au niveau des organes hématopoïétiques, fait que nous n'avons pas vérifié. Il n'est pas impossible qu'une faible quantité de fer ait également traversé le placenta comme l'ont montré HANSARD et al. (I962), mais nous n'avons trouvé aucun accroissement apparent des dépôts de fer intra-utérins, notamment du foetus entier ou du foie foetal. Ceci rejoint donc les observations antérieures réalisées sur plusieurs espèces après l'administration sous diverses formes de supplément de fer aux femelles gestantes (POND, I960; CHANEY et BARNHART, I963; MILLER, I 964). Or, plusieurs expériences réalisées à l'aide de fer radioactif, avaient montré que le fer injecté pouvait franchir rapidement la barrière placentaire et se fixer dans les productions utérines à des taux variables suivant le stade de gestation. Ce fut le cas de l'Homme (Pommerenke et $a l$., I942) du Lapin (Bo'thwel. et al., 
I958), de la Brebis (Hansard et Hoskins, I964) et de la Truie (Hansard et Hoskins, I962, I 964). Ceci se trouve confirmé dans notre expérience par le fait que l'on observe à la parturition beaucoup plus de fer dans les fotus que dans le placenta ou la paroi utérine en dépit d'une circulation à contre-gradient. Il convient toutefois de noter que les modalités du transfert du fer peuvent différer entre les espèces, suivant les particularités propres à chaque type de placentation. On sait également que le fer qui pénètre dans les productions utérines provient principalement, sinon exclusivement, des sidérophilines plasmatiques (BOTHWELI, et al., I958) et non des hématies maternelles comme le pensait STANDER (I94I) ou d'une sécrétion utérine (WISLOCK et Dempsey, I946). On peut donc penser que l'enrichissement en fer du fotus n'est possible que si la sidérémie maternelle s'accroît. Bien que nous ne l'ayons pas encore vérifié, ceci ne semble pas s'être produit ici.

On peut également supposer que le transfert de la mère au fœtus, est limité par le taux de siclérophiline plasmatique fotal et la capacité de fixation en fer de cette sidérophiline. Par ailleurs, il est vraisemblable que les possibilités de stockage hépatique du fœetus sont faibles (tabl. 5) et que les besoins stricts en fer des fœtus ont pu être couverts par l'apport de notre régime le plus pauvre en cet élément (75 p.p.m.). Ce n'est effectivement que dans le cas de carences maternelles profondes que l'influence bénéfique d'un supplément de fer apparaît (Ny,ANDER, I953; BoTHWELI, et $a l .$, I958; Morgan, I96I). Or Rydberg et $a l$., (I959) avaient signalé qu'une simple dose de $500 \mathrm{mg}$ de fer injectée à la mère 2 semaines avant la parturition suffisait à couvrir les besoins de l'hématopoïèse fœtale. Dans le cas présent, les doses que nous avons utilisées $(4 \mathrm{~g})$ ont peut-être été excessives et ont sans doute été utilisées davantage par la mère que par ses fotus.

Le second point concernait l'enrichissement éventuel en fer du lait de Truie Il convient ici d'être plus nuancé : l'enrichissement est possible, mais il ne paraît pas suffisant. Bien qu'à la suite de nombreux échecs (HAmilton et al., I930; VENs et al., I947, Hooks et $a l .$, I963, Veum et $a l .$, I965) cet enrichissement ait été jugé problématique, d'autres que nous l'avaient déjà observé (CHANEY et BARNHART, I963 ; HANSARD et $a l .$, I964).

L'amélioration d'environ 50 p. I00 que nous avons constatée est du même ordre que celle rapportée par CHANEY et BARNHART, (I963) et le fait d'avoir employé un fer injectable, ce qui permet de surmonter le défaut d'absorption de la paroi intestinale, a pu faciliter le phénomène. Mais pour être efficace dans la prévention de l'anémie, l'accroissement de la teneur en fer du lait aurait dî̀ être 3 à 4 fois plus grand de façon à couvrir le besoin minimum quotidien du porcelet estimé à environ $7 \mathrm{~m} g$ par VENN et al., (I947). Dans notre expérience, le lait des truies supplémentées n'apportait pas plus de $r, 5 \mathrm{mg}$ de fer par jour à chaque porcelet. Il n'y a rien d'étonnant à ce que ces animaux, qui ne bénéficiaient d'aucune autre source de fer, aient été anémiques pour la plupart et que le lot supplémenté ne se soit pas mieux comporté à cet égard que les autres lots, ce qui rejoint les observations des auteurs précédents.

Quoi qu'il en soit, l'administration de fer dextrane hydrogéné par la voie intramusculaire aux truies n'a pas eu l'effet escompté et n'a pas été plus efficace au cours de la lactation qu'elle ne l'avait été au cours de la gestation. C'est ce qu'avaient également observé Ruthedge et 'TEAGue, (I959) et Buysse (I962).

Un point demeure cependant acquis : c'est que, dans un cas comme dans l'autre, le transfert placentaire ou mammaire du fer injecté à la Truie est possible et n'est 
donc pas directement mis en cause. Mais il conviendrait maintenant d'en mieux connaître les modalités et notamment le rôle que jouent certaines protéines plasmatiques maternelles dans ce domaine. Le même problème se pose d'ailleurs pour le Porcelet, pour lequel l'apport direct de fer pendant l'allaitement reste actuellement la seule solution efficace techniquement et économiquement qui permette de pallier l'anémie post-natale.

Rę̧u pour publication en décembre 1967.

\section{REMERCIEMENTS}

Nous adressons nos sincères remerciements à la Société SoveTaL, dont la contribution matérielle nous a aidé à réaliser cette étude.

\section{SUMMARY}

\section{UTILIZATION OF AN INJECTABIE IIYDROGEN IRON DEXTRAN COMPOUND}

BY THE SOW DURING PREGNANCY AND LACTATION

The effects of an injectable hydrogen iron dextran compound on intra-uterine iron concentration and milk iron content were studied on 30 gilts alloted in three groups.

One group (T) was intramuscularly injected with $4 \mathrm{mg}$ of iron at days 55 and $\mathrm{I}$ Io of pregnancy. The two other groups ( $S$ and 0 ) were used as controls, one (S) being supplemented with dietary $\mathrm{SO}_{4} \mathrm{Fe}$.

At day to of lactation, half the animals of each group were injected with $4 \mathrm{mg}$ of iron.

$\mathrm{I}$ - There was a considerable increase in the amounts of iron stored in the liver. An increase of smaller magnitude was noticed in the uterine wall, but no alteration in the iron content of any fetal tissue was observed either at day 60 or at birth.

Therefore, it may be thought either that injected iron cannot pass the placental barrier, or that the fetus is not able to store iron.

II - The iron content of milk significantly increased from 1.6 to $2.2 \mathrm{mg}$ per $\mathrm{kg}$, though not sufficiently to meet the requirements of the piglet.

III - Litter performances at farrowing and at weaning were identical in the three groups. The piglets were found anemic whatever the treatment of the sows. This leads us to the view that high rates of iron injected to sows cannot improve the protection against anemia of the progeny, either during pregnancy or during lactation.

\section{RÉFÉRENCES BIBLIOGRAPHIQUES}

Ai.fFax R., Bejambes M., i956. Dosage simultané du fer et du cuivre dans le lait et les produits laitiers. Ann. Tech. Agric., 5, 6r $9-634$.

Bothwelt, T. M., Pribilla W. F., Mebust W., Finch C. A., 1958. Iron metabolism in the pregnant rabbit ; iron transport across the placenta. Amer. J. Physiol., 193, 61 5-622.

Buysse F., 1962. Anaemia in piglets (holland). Tschr. diergeneesk, 8\%, 1423-1 439.

Chaney C. M., Barnhart C. E., I963. Effect of iron supplementation of sow rations on the prevention of baby pig anaemia. J. Nutr., 81, 187 -192.

Duncan D. B., 1955. Multiple range and multiple F tests. Biometrics, 11, I-42.

Elveujem C. A., Herrin R. C., Hart E. B., I927. Iron in nutrition. III. The effects of diet on the iron content of milk. J. Biol. Chem., 71, 255-262. 
Hamilto: T. S., Hunt (i. E., Mrtchel. I. N., Carrolt, W. F., r930. The production and cure of nutritional anaemia in suckling pigs. J. Agric. Res., 40, 927-935.

Henri K., 1945. Technique de dosage du fer sanguin. Ann. Biol. Chim, 3, 101-105.

Hooks R. D., Hays V. W., Spekr V. C., ICCAIL J. F., 1963. Control of baby pig anaemia by feeding high levels of iron to lactating sows. J. inim. Sci., 22, 1 1 22 (Abstr.).

Hoskiss F. H., Hansaru) S. I., so62. J'acental transfer and absorption of iron in swine J. Anim. Sci., 21. 1024-1025 (Abstr.).

Hoskiss F. H., Hansaro S. I., rg6. I'lacental transfer and fetal tissue iron utilization in sheep. $J$. Nutr., 83, 10-14.

Hoskixi F. H., Hansarn S. J., ig6t. P'lacental transfer of iron as a function of grestation agre. Proc. Soc. Exp. Biol. and Med., 116, 7-11.

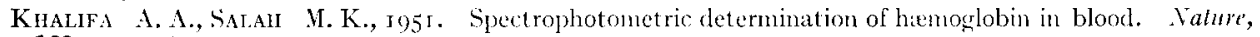
168, y) $5-016$.

I.e Dunnci J., r 967 . Anémie du porcelet sous la mère : essais de supplénentation en fer. Résultats non publiés $\left(C . X^{\prime} . R . Z\right.$. .

Linkexheimer W. H., 1962. Placental transfer of iron. J. Anim. Sci., 21, 1026 (Abstr.).

Mileer l: R., ig63. I'larental and mammary transfer of iron from iron chelates in the sow's diet. 7 . Anim. Sici, 22, 845 (.1bstr.).

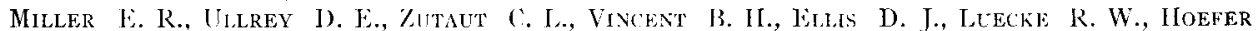
J. A., I 904 . Supplementation of sows lactation diet with ferrous fumarate. J. Anim. Sci., 23, 884 (Absir.).

Morgax E. H., г $96 \mathrm{I}$. 'T'ransfer of iron from the pregnant and lactating rat to fetus and young. $/ . P h y^{-}$ siol., 158. $573-586$.

Nylanier G., 1953. On the placental tramsfer of iron. An experimental sturly in the rat. Aca Physiol. Scant. 29. suppl. 107, 1-105.

Pommereske W. T., Hain P. F., Bil.E W. T., BAlfour W. M., Ig42. Transmission of radioartive iron to the human fetus. Amer. J. Physiol, 137, 164-1 70.

Pond W. G., 1960. Liffect of oral and parenteral iron administration to suchling pigs and to sows. Proc. Cornell Vitir. Cont., s[-95.

Rutledge E. A., Teacle H. S., 195\%. In (hio .1gric. Exp. Sta. Anim. Sci. Mimeo no in 4 , p. 28.

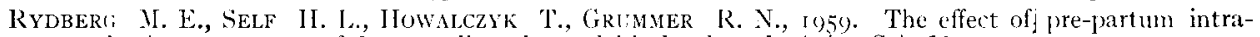
muscular iron treatment of dams on litter hemoglobin levels. J. Lnim. Sci., 18, 415-4Ii).

Strander II. J., ig4r. Williams (Mhsletrics, ed. 8, New Jork, Appleton Century Crofts inc.

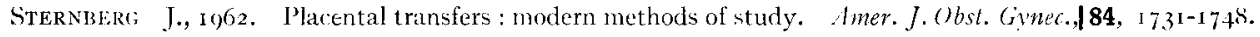

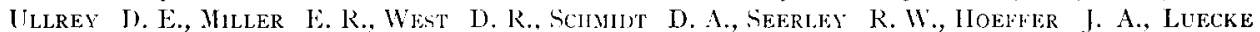
R. W., I959. Oral and parenteral administation of iron in the prevention and treatment of baby pig anaemia. J. Inim. Sci., 18, 25(1)-263.

VenN J. A. J., MCCANCE R. A., Wibmowson F. II., 1947. Iron metabolism in piglet andemia. J. Comp. Pathol. Therapeutics, 57, 31 4-325.

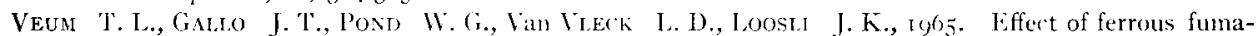
rate in the lactation diet on sow milk iron, piy henowlobin and weight grain. J. Inim. Sci., 24, 1 169-1 I 73 .

Wislock G. B., Dearser Ji. W., 1946. Histochemical ractions of the placenta of the pig. Amer. J. Inat., 78, $181-225$. 